\title{
Tardive dyskinesia presenting as severe dysphagia
}

\author{
R P Gregory, P T Smith, P Rudge
}

\begin{abstract}
A case of tardive dyskinesia presenting with severe dysphagia as the predominant feature is described. Diagnosis was delayed because clinically apparent orofacial dyskinetic movements were minimal. The symptoms resolved following the cessation of neuroleptic medication.
\end{abstract}

(F Neurol Neurosurg Psychiatry 1992;55:1203-1204)

It is well recognised that dyskinetic involuntary movements may be induced by neuroleptic medication. ${ }^{12}$ These usually consist of repetitive choreiform movements in an oral-lingualbuccal distribution. Only if the movements are severe is dysphagia recognised as a significant problem, ${ }^{3}$ and this is usually due to abnormal tongue movements. ${ }^{4}$ These prevent the adequate formation of a food bolus and its coordinated passage into the pharynx. We report a patient with tardive dyskinesia (TD) presenting as severe dysphagia, where the absence of clinically obvious involuntary movements delayed the diagnosis and subsequent successful therapy. This has not previously been described.

\section{Case report}

A 48 year old Asian nurse presented with a six

\section{National Hospital for Neurology and Lourosurgery, R P Gregory P T Smith \\ Correspondence to: Dr Gregory, Neurology Department, Princess Wroughton, Swindon SN4 OQJ, UK \\ Received 29 January 1992 Accepted 24 April 1992}

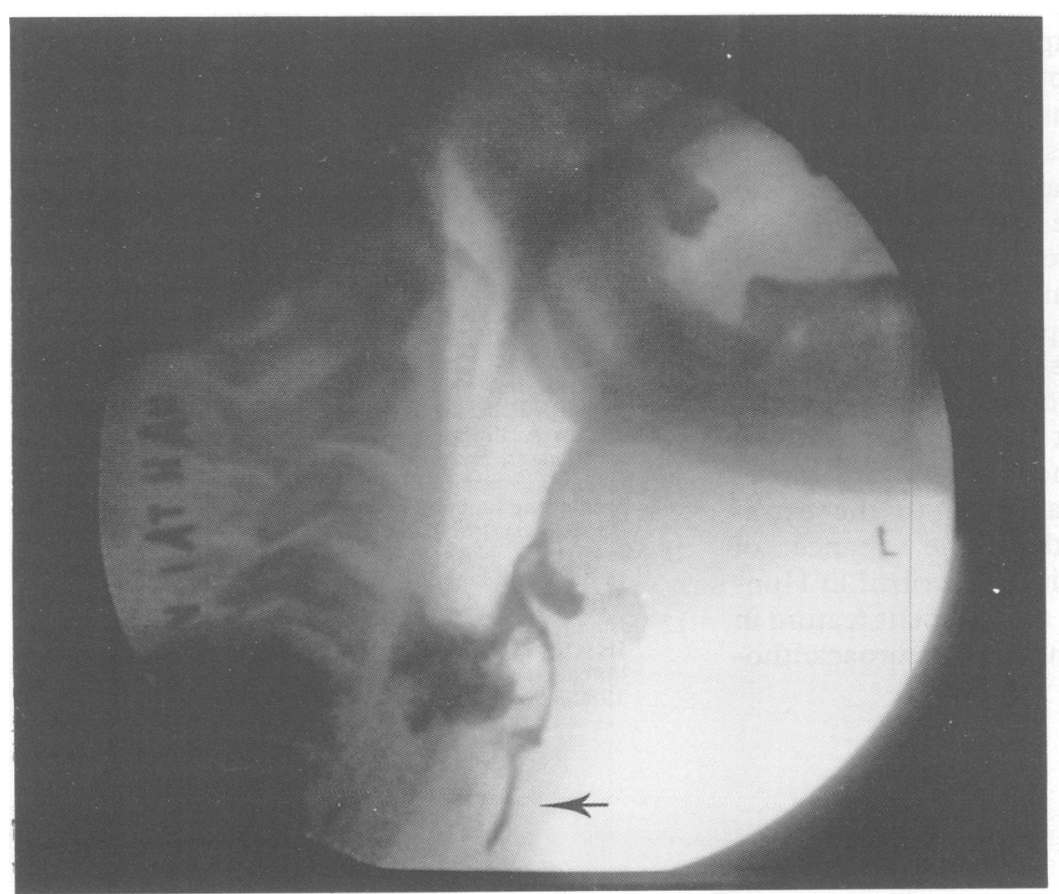

Figure Video-swallow showing pooling of liquid in pharyngeal sinuses and tracheal aspiration (arrow) after completion of swallow. year history of insidiously progressive swallowing difficulties. She first noticed that solids lodged behind her sternal notch and that this was helped by swallowing water. Subsequently she had regurgitation of fluids with choking attacks. In the previous six months she was only able to take a semi-solid diet with great difficulty and had lost 12 kilograms in weight. She was otherwise well with no other relevant symptoms or family history. Initial neurological and otolaryngological assessment including endoscopies and routine barium meal examinations at the referring hospital was unrewarding.

On examination she had occasional tics of the face without dyskinetic movements of the tongue or pharynx. She could maintain tongue protrusion and voluntary movements were normal. There was no dysarthria. Neurological and general examination were otherwise normal. Video-fluoroscopic barium swallow revealed an abnormal oral stage with disorganised tongue movements, pooling in the pharyngeal sinuses, and no significant laryngeal elevation or closure of the vestibule. Silent aspiration occurred with solids and liquids, which were not sensed or cleared (figure). The following investigations were normal or negative: full blood count, ESR, thick film for acanthocytes, biochemical screen, $\mathrm{CK}$, copper studies, acetylcholine receptor antibodies, CSF studies, CT brain scan, concentric needle and single fibre EMG, nerve conduction studies and evoked potentials. In view of the substantial risk of aspiration a further otolaryngological opinion was sought. She subsequently had a cricopharyngeal myotomy and epiglottic plication in an attempt to protect her laryngeal inlet. ${ }^{5}$ Muscle biopsies taken at the time of the procedure were normal. Unfortunately she gained no benefit and further videofluoroscopic assessment demonstrated continuing aspiration. Nutrition was maintained via a nasogastric tube.

Further information regarding her past medical history was then revealed. Ten years previously she had developed a manic-depressive psychosis which had then been difficult to control because of poor compliance. For six years she had received monthly depot flupenthixol injections in standard doses. She initially had jaw spasms and torticollis which rapidly resolved. Following this she took oral chlorpromazine in variable doses, until four months before presentation when lithium was substituted. Serum lithium levels were maintained within the therapeutic range. The possibility of TD as a cause of her dysphagia was then considered. Apart from occasional blinks, 
there were no abnormal facial movements. She was able to maintain tongue protrusion, but there were occasional movements of the tongue in the floor of the mouth. The video swallow was reviewed and the involuntary movements of the tongue at rest were confirmed. It became clear that much of her abnormal swallow could be explained on the basis of involuntary dyskinetic and dystonic movements of the tongue, pharynx, and larynx. Her dysphagia had deteriorated after withdrawal of the chlorpromazine, and so this was reintroduced for a trial period. This coincided with an improved swallow both subjectively and radiographically, but some aspiration of liquid continued. The nasogastric tube was successfully removed. Chlorpromazine was then substituted with benzhexol, increasing to a maximum of $21 \mathrm{mg}$ per day. Although this maintained the improvement in her swallow, it precipitated prominent oro-facial dyskinesia and was withdrawn by the patient.

She remained off all medication for 10 months and was then reviewed. The clinically apparent dyskinetic tongue movements were still present but symptomatically her swallow has dramatically improved. She was able to drink fluids in large volumes without difficulty and had regained all the weight she had previously lost. Her video swallow now showed much less dyskinetic movement, with improved laryngeal elevation and coordinated swallow. There was minimal aspiration of fluids but this was now successfully cleared on completion of the swallow.

\section{Discussion}

Although this case is atypical, the diagnosis of TD appears secure. Normal tongue protrusion occurs in $50 \%$ of patients with TD, and involuntary tongue movements within the buccal cavity, may be the only sign in "mild" cases. ${ }^{6}$ Her gradual improvement after withdrawal of all neuroleptic medication made alternative diagnoses unlikely. Dysphagia in movement disorders has received scant attention in the literature, ${ }^{7}$ although patients with Parkinson's disease have been assessed in some detail. ${ }^{4}$ They often have delayed initiation of the swallow, and combined with reduced laryngeal closure and pharyngeal peristalsis, aspiration may occur. As in our patient, it is not unusual to find chronic aspiration on radiographic studies without coughing or external signs of aspiration. When involuntary movements are present in addition to the extrapyramidal disorder, then the chance of dysphagia increases. This is described in Huntington's disease ${ }^{8}$ and is a prominent feature in more rare conditions such as neuroacanthocytosis. ${ }^{9}$
Previously there have only been occasional reports of patients with drug induced tardive dyskinesia and dystonia resulting in dysphagia. A study in a long stay psychiatric institution used cinefluorography to study the swallow of 35 chronic schizophrenic patients without obvious symptoms of dysphagia, although the presence of TD was not commented upon. ${ }^{10}$ Twelve had significant swallowing abnormalities often with pooling in the valleculae and piriform sinus and 2 patients had tracheal aspiration. The authors proposed this as a likely cause of sudden death in this population. Another study examined three patients with TD using cinefluorography. ${ }^{11}$ The abnormal tongue movements were described but abnormalities in the other bulbar musculature were not mentioned. More recently a patient with bulbar and cervical dystonia provoked by chlorpheniramine was reported. ${ }^{12}$ The dysphagia was due to inadequate lip closure with severely impaired tongue control resulting in food falling prematurely into the pharynx. Unlike our patient, the pharyngeal stage of the swallow was normal. When involuntary movements are principally confined to the larynx it is easy to overlook dyskinesia as a possible cause. Routine barium meal examinations and endoscopic procedures will not identify abnormalities in these patients and video-fluorography is required. ${ }^{34}$

This apparently unique case emphasises the need to consider dyskinesia as a cause of dysphagia, even in the absence of more obvious abnormal movements, and to be aware of the possibility of silent aspiration in cases of tardive dyskinesia and other patients with movement disorders.

1 Kane JM, Smith JM. Tardive dyskinesia. Arch Gen Psychiatry 1982;39:473-81.

Kang UJ, Burke RE, Fahn S. Tardive dystonia. $A d v$ Neurology 1988;50:415-29.

3 Wiles CM. Neurogenic dysphagia. $\mathcal{f}$ Neurol Neurosurg Psychiatry 1991;54:1037-9.

4 Logemann JA. Dysphagia in movement disorders. Adv Neurology 1988;49:307-316.

5 Brookes GB, McKelvie P. Epiglottopexy: a new surgical technique to prevent intractable aspiration. Annal Roy Col Surg Eng 1983;65:293-6.

$6 \mathrm{Pi}$ EH, Simpson GM. Tardive dyskinesia and abnormal tongue movements. Brit f Psychiatry 1981;139:526-8.

7 Massengill R, Nashold B. Cinefluorographic evaluation of swallowing in patients with involuntary movements. swallowing in patients with
Confin Neurol 1969;31:269-72.

8 Leopold NA, Kagel MC. Dysphagia in Huntington's disease. Arch Neurol 1985;42:57-60.

9 Hardie RJ, Pullen HWH, Harding AE, et al. Neuroacanthocytosis. A clinical, haematological, and pathological study of 19 cases. Brain 1991;114:13-50

10 Hussar A, Bragg D. The effect of chlorpromazine on the swallowing function inchronic schizophrenic patients. $\mathrm{Am}$ f Psychiatry 1969;126:570-3.

11 Massengill R, Nashold B. A swallowing disorder denoted in tardive dsykinesia patients. Acta oto-laryngologica 1969;68:457-8.

12 Bosma J, Geoffrey V, Thach BT, Weiffenbach JM, Kavanagh $J \mathrm{~F}$, Orr W. A pattern of medication induced persistent bulbar and cervical dystonia. Int $\mathcal{f}$ Orofac Myol 1982;8:5-19. 